\title{
LES USAGES DES ARCHIVES DE TÉLÉVISION
}

\author{
Nabil Aliouane ${ }^{1}$ \\ Il y a des gens qui ont une bibliothèque, comme les eunuques \\ ont un harem. \\ Victor Hugo
}

Le bon mot de Victor Hugo, s'il prête à sourire, ne pointe pas moins fortement la question de l'usage des archives et dévoile de nombreuses réalités : de la mythique bibliothèque d'Alexandrie aux gigantesques bases de données informatiques, une seule constante: l'esprit de conservation. L'usage des archives dépend avant tout de la conception qu'on en a ; de fait tout domaine peut faire l'objet d'un archivage: les différents musées, des plus loufoques aux plus classiques, en sont un bon exemple.

La télévision n'échappe pas à cette constante, et depuis les débuts du média, on a cherché à conserver, plus ou moins bien, les émissions diffusées. Si tout n'a pas été conservé, si des émissions entières, des milliers d'heures ont disparu, ces pertes sont minimes, en comparaison d'autres médias, tant du point de vue qualitatif que quantitatif. Ceci est en grande partie dû au fait que la télévision est, à ses premières heures, une affaire d'État; la création télévisuelle est donc, pour un temps, patrimoine national. Cet état de grâce va

1 Chercheur au Centre de Recherches sur les Médiations (CREM) de l'Université de Metz. 
permettre de constituer rapidement une banque de données, un stock d'émissions conséquent. De fait, il semble plus pertinent, concernant la télévision, de parler de stock ou de base de données, en raison de la mobilisation permanente de ce stock, car le terme d'archives induit une certaine statique, et s'il y a bien une caractéristique propre à l'archivage de télévision, c'est au contraire ce mouvement permanent.

Nous assistons depuis quelques années à un fort développement d'émissions prenant pour thème la télévision, ce que nous nommons les émissions prismatiques afin d'éviter l'écueil réflexif et rendre pleinement compte de ce phénomène. Ces émissions, qui ont pour caractéristique principale d'être fortement consommatrices d'archives de télévision, ne forment pas un miroir (réflexif) mais un prisme, c'est-à-dire un espace de transfert qui va donner une vision décalée (au sens propre et au figuré) et/ou décomposée de la source.

Cette pratique n'est pas nouvelle. Dès les débuts de la télévision, on a recyclé les images, on a consacré des émissions entières à revisiter le passé encore récent de la télévision. Rien d'étonnant à cela; les impératifs de flux et de vitesse sont tels à la télévision, que l'image, l'émission prend le statut d'archives dès la fin de sa première diffusion. Dès lors, elle fait partie du stock et peut être mobilisée à tout moment : le jour même, le lendemain, la semaine d'après, dans 10 ans... L'émission prismatique va construire son discours en utilisant le stock d'émissions disponibles par le biais de la citation. Les modalités et formes citationnelles sont nombreuses et tout, dans l'émission mobilisée, peut faire l'objet d'une citation : de la microséquence à l'émission entière. Si le flux de la première diffusion préserve l'unité de l'œuvre, le stock fait éclater cette unité et permet un découpage quasi illimité de l'œuvre selon les modalités de citation et les effets recherchés.

\section{A quoi servent les archives?}

Si les objectifs affichés varient selon que l'émission s'inscrit dans une logique ludique ou informative, voire les deux, il est cependant une constante à toutes les déclarations d'intention. Toutes évoquent le retour, l'arrêt ; il va s'agir de revenir sur le passé, de revoir des images, d'arrêter le flux télévisuel, de redécouvrir une période, de revisiter une œuvre... Ceci dans le but de : 
- mieux comprendre le monde. «En gros, il y a les JT, les débats et les longs reportages. Nous arrivons le samedi, pour dire : "Voilà ce qui s'est vraiment passé d'important cette semaine, et nous allons essayer de vous expliquer pourquoi" " ${ }^{1}$ (B. Benyamin à propos du magazine Face à l'image diffusé sur France 2, le samedi à 17H50);

- mieux comprendre la télévision, les médias. "Vous êtes perdu dans la jungle des médias ? Le trop plein d'informations vous noie ? +Clair, le magazine de tous les médias et des coulisses de l'information, vous aide à y voir plus clair...»² (présentation du magazine +Clair par la chaîne) ;

- critiquer les modalités de fabrication des images. «Chaque dimanche, la télévision fait son autocritique sur France 5. Daniel Schneidermann et son équipe de chroniqueurs auscultent le petit écran sous toutes ses formes et dans tous ses excès, et analysent le foisonnement et l'influence des images télévisuelles dans notre société» ${ }^{3}$ (à propos d'Arrêt sur images);

- mieux connaître les stars du petit écran. «Vous rêviez de voir vos stars préférées faire la popote, prendre leur petit déjeuner en famille le cheveu en bataille ou offrir leur corps entièrement nu à la mer ? C'est aujourd'hui chose possible grâce à Stars intimes, le nouveau rendez-vous de M6... Nous voulions faire oublier les projecteurs et le vernis d'un plateau télé. L'intérêt était de voir des têtes connues en tenue décontractée et sans maquillage» ${ }^{4}$.

- ou encore passer un bon moment en revoyant des images qui ont pu marquer sa jeunesse, l'année écoulée ou la journée d'hier. «Rien n'a échappé au grand maître Arthur et à son équipe de cuisiniers documentalistes du rire. Ils ont choisi pour ce nouveau grand bêtisier des images inédites, toujours aussi surprenantes, amusantes ou émouvantes, que leurs acteurs ou des témoins viennent commenter sur le plateau. Tous, simples anonymes, comédiens, animateurs à la télévision, journalistes ou animaux... feront la joie des petits et des grands amateurs d'inattendu, de loufoque et d'humour» ${ }^{5}$. (à propos de l'émission 120 minutes de bonheur).

1 Télé Loisirs, $\mathrm{n}^{\circ}$ 916, 20-26 septembre 2003.

2 http://www.canalplus.fr/info/plusclair/

3 http://www.france5.fr/asi/

4 Télé Loisirs, $n^{\circ} 917$ du 27 septembre - 03 octobre 2003.

5 Télé Loisirs , nº 883 du 26 janvier - 01 février 2003. 


\section{La répétition comme acte de discours}

Léo Apostel définit un texte comme «une séquence d'actes de discours qui peut être considérée en elle-même comme un acte de discours unifié»', ce qui signifie, comme le postule Frédéric Nef «qu'il existe des macro-actes de langage qui subsument les microactes de langage $»^{2}$. Il nous faut donc envisager le texte à la fois au niveau macro-textuel et micro-textuel.

L'utilisation des archives de télévision est une pratique (prismatique) de monstration télévisuelle complexe, construite sur des actes de discours ; ces actes de discours doivent être envisagés à la fois au niveau macro- et micro-textuel ${ }^{3}$.

Au niveau macro-textuel : nous retiendrons deux catégories principales d'actes de discours :

- Promettre : cette catégorie est déjà citée par Austin (commissif) et Searle. Tous deux en donnent la définition suivante : «(actes) dont le but est d'obliger le locuteur à adopter une certaine conduite future». Selon le cas, l'émission prismatique porte la promesse, par la voix de son animateur, d'un retour sur image, d'une meilleure compréhension du média, d'un droit à la critique... ou tout simplement la promesse de passer un moment agréable.

- Référer à : après la promesse, c'est le fondement des émissions prismatiques, si bien que l'on peut dire que s'il n'y a pas référence, donc mobilisation des archives, il n'y a pas d'émission prismatique.

Nous ne retenons que deux catégories au niveau macro-textuel car elles semblent être les seules capables d'englober l'ensemble des émissions. Par contre au niveau micro-textuel, les actes sont beaucoup plus nombreux et leur apparition est variable, tout est fonction des objectifs affichés (promis) et cachés. Il paraît peu efficace d'essayer d'en dresser une liste exhaustive, c'est à l'analyste de les repérer au

1 D'après J.-M. ADAM, Éléments de linguistique textuelle, Bruxelles-Liège, Mardaga, 1990, p. 103.

2 D'après C. Kerbrat-Orecchioni, Les actes de langage dans le discours, Paris, Nathan, 2001, p. 158.

3 Nous précisons que nous ne cherchons pas à référencer tous les actes de discours mais à proposer, à la manière d'Austin, des catégories générales pouvant englober un maximum d'émissions prismatiques, voire toutes. 
sein de son corpus ; pour autant, nous pouvons citer les plus répandus dans les émissions prismatiques qui sont :

- faire rire ;

- se moquer ;

- rappeler ;

- informer d'avantage ;

- expliquer ;

- commenter ;

- critiquer ;

- honorer ;

- admirer.

\section{Tout se répète}

Cette pratique ne se limite plus aux seules émissions dédiées, mais investit l'ensemble du média. A cela trois grandes raisons : sémiotiques et esthétiques, culturelles et économiques.

\section{a. Raisons sémiotiques et esthétiques}

La télévision, plus que tout autre média, a consacré la sérialité, non seulement comme une forme d'écriture à part entière, mais comme une esthétique qui régit l'ensemble du média, et non plus seulement les formes fictionnelles. La télévision fonctionne essentiellement selon une logique sérielle : organisation cyclique des programmes, des schémas d'émissions, rendez-vous fixés au lendemain par les animateurs... L'esthétique de la télévision est donc essentiellement une esthétique de la répétition fondée sur deux grands principes : la symbolique et le plaisir.

- L'acte de répétition réduit considérablement la portée informationnelle de l'émission, pour privilégier au contraire son caractère rituel et symbolique. Si la meilleure communication est à taux d'information zéro, la sérialité offre ce confort d'une émission, d'un schéma connu dans lequel le téléspectateur n'a pas à chercher d'informations, donc de choses nouvelles, puisque tout se répète. Le téléspectateur attend impatiemment que les acteurs répètent inlassablement les mêmes gestes, prononcent les mêmes mots : «C'est votre dernier mot ?» (Qui veut gagner des millions), «Correct !» (Le 
maillon faible), «Le rideau, le rideau !» (Le Bigbil), «Vous regardez toujours trop la télévision, bonsoir !» (Les Guignols de l'info), «L'actualité c'est vous qui la vivez, c'est nous qui en vivons» ( 7 jours au Groland). Ce sont ces mots clés associés aux moments phares qui structurent l'émission et créent le sens.

- La sérialité est inséparable de la notion de plaisir. «Une série est d'abord, pour un téléspectateur amateur, l'assouvissement du plaisir de la répétition : celui-ci est bien entendu aussi celui de la variation, son compagnon indispensable» ${ }^{1}$. Le téléspectateur, comme le jeune enfant (c'est notamment sur ce point que les études sur la réception peuvent nous éclairer davantage), éprouve un réel plaisir dans la répétition de ces gestes et paroles. Ce plaisir est lié à la fois à la sécurité qu' offre la sérialité (tout est connu) et à l'anticipation qu'elle permet, comme pour la série fictionnelle. Il est sécurisant de retrouver les personnages inchangés, de connaître par avance leur réaction face à une situation, en résumé, d'avoir l'impression que rien n'a changé. Mais ce plaisir est aussi fortement lié au sentiment de communion que procure la sérialité. Tous les acteurs : animateurs, téléspectateurs, participants (qui sont aussi des téléspectateurs) se "rassemblent" pour accomplir "ensemble" le même rituel. La sérialité est à la base de la puissance de la télévision, aucun média n'offre avec. autant de force ce sentiment de communion ${ }^{2}$. C'est la répétition de ces actes rituels et symboliques qui permet au téléspectateur de se constituer comme membre d'une communauté de spectateurs. Ainsi, les archives peuvent être mobilisées autant de fois que nécessaire, sans pour autant lasser le regard. On peut citer pour l'exemple l'émission Les enfants de la télé qui pendant plus d'une saison terminait systématiquement sur un extrait des adieux à la nation du Président Giscard d'Estaing et son fameux «Au revoir».

1 J.-P. EsquenAzI, "L'inventivité à la chaîne", in M.E.I., nº 6 : Télévision, la part de l'art, G. Delavaud (dir.), Paris, 2002, p. 105.

${ }^{2}$ Le média le plus proche de ce modèle est la radio, mais la télévision dispose en plus du pouvoir de captation de l'image. L'Internet propose jusqu'à présent un modèle de communauté différent, c'est l'acte de naviguer sur le web qui lie les internautes, pas le site qu'ils visitent. 


\section{b. Raisons culturelles}

Il est loin le temps où la télévision était considérée comme un objet de foire, un "monstre" hybride entre le cinéma et la radio, ne possédant ni la beauté de l'un, ni le talent de l'autre. C'est pourtant ce mélange bâtard qui va donner toute sa force au média, lorsque la technique a progressé : qualité et taille de l'image, baisse du prix des récepteurs, qualité de la transmission (notamment du direct), essor progressif de la zone de couverture jusqu'à atteindre l'ensemble du territoire, développement des liaisons intercontinentales... La télévision a su transformer ses "défauts" en qualités et ainsi allier la "vitesse" de la radio à "l'esthétique" du cinéma. La télévision tire cette légitimité de la masse de gens qu'elle attire ; elle devient dès lors un passage obligé pour les intellectuels et artistes cherchant à promouvoir leurs œuvres. Cette légitimité rapidement acquise a donné une réelle valeur à l'image de télévision, en lui attribuant un réel pouvoir testimonial. Cette valeur de l'image a aussi fait des archives de télévision une source légitime pour les historiens et autres scientifiques. Cela a également permis à la télévision de prendre en charge, elle-même, le travail de mémoire, d'écrire progressivement l' histoire du média avec un temps de recul très court, de consacrer ses moments clés, de célébrer ses acteurs et ses productions. Elle consacre aussi ses historiens : comment imaginer une émission souvenir sans la présence de la mémoire vivante de la télévision, Pierre Tchernia, devenu l'icône de cette télévision du passé. Pourtant, la télévision n'a guère plus de 60 ans, mais la vitesse et les mutations constantes du média "vieillissent" les images et les perceptions.

\section{c. Raisons économiques}

Nous n'oublions pas que la télévision est avant tout une industrie culturelle; et comme toute industrie, sa motivation principale est la réalisation de profits. Nous entendons par industrie culturelle le fait que des biens culturels soient reproduits mécaniquement et écoulés de manière massive sur un marché. L'industrie culturelle se caractérise aussi par la mise en place de formats visant à rentabiliser la production en baissant les coûts et par la salarisation de tous les 
acteurs de la chaîne de création. A la différence du cinéma et de l'édition où le client achète sa place pour une séance ou son livre, la télévision (hertzienne) ne vend pas directement son produit au téléspectateur car quelqu'un paie pour lui. En fait, la principale source de revenus d'une chaîne vient de la publicité ; l'activité principale d'une chaîne, en termes de priorité économique, est donc de vendre son espace publicitaire. En caricaturant, on pourrait dire que les différents programmes ne sont là que pour attirer le client vers la publicité qui, elle, doit conduire à l'achat du produit. D'où l'intérêt de la chaîne à construire ses programmes autour d'une grille routinière, car elle permet de s'assurer d'un nombre constant de téléspectateurs. La sérialité investit tous les genres sous la "pression" économique des annonceurs ; les programmes existent grâce aux financements des annonceurs, qui eux-mêmes investissent parce que ce programme leur permet d'atteindre leurs clients.

Pour la chaîne, les coûts sont donc relativement fixes, et comme pour le cinéma, une fois que les coûts de production de l'original sont amortis, les coûts de reproduction ou de diffusion sont insignifiants. Les médias fonctionnant selon une logique éditoriale constituent leur marge bénéficiaire sur le nombre et la durée. Schématiquement : plus un film réalisera d'entrées, plus longtemps il restera à l'affiche, plus il réalisera de profits. Cette stratégie n'est pas applicable pour les médias de flot; on ne peut pas diffuser tous les jours le même programme puisque les téléspectateurs sont sensiblement les mêmes. Pour les médias de flot, la diffusion est éphémère. En télévision, cette difficulté est contournée par l'esthétique développée, qui est essentiellement une esthétique de la sérialité (pour les programmes fictionnels et non fictionnels), ce qui fait que le téléspectateur est habitué à revoir les mêmes images, voire les mêmes émissions ou séries.

Les années 80 ont vu émerger les compilations dans l'industrie du disque, les coffrets collector dans l'industrie du cinéma et la multiplication des émissions prismatiques à la télévision. Ces phénomènes sont très liés et partent du même principe : le recyclage d'un succès déjà éprouvé. Les émissions prismatiques assurent à la chaîne une audience importante pour un investissement minime, surtout s'il s'agit d'une émission best of réunissant les meilleurs moments de la saison. Les chaînes y trouvent le moyen de recycler des émissions de flot qui, auparavant, devaient être rentables lors de leur unique diffusion. Ainsi, elles modifient même l'acception de 
produits de flux et produits de stock, tout programme pouvant désormais être réutilisé selon différentes modalités. Le matériau de ces émissions est le plus souvent disponible dans les archives des chaînes et ne demande qu'à être recyclé. Les risques sont minimes, tant au niveau du coût de production dérisoire, qu'en termes d'audience, puisque l'émission est constituée de moments déjà vus et appréciés des téléspectateurs. L'émission prismatique utilise le succès de/des émissions qu'elle mobilise. En cela, elle rejoint le modèle de la série qui offre une sécurité liée à la connaissance préalable du public, à son désir de revoir les "meilleurs moments" des émissions déjà diffusées, lié (nous l'avons vu) au plaisir de la répétition.

Les récentes évolutions des grilles de programmes montrent qu'il n'y a aucun doute que cette pratique va s'étendre à l'ensemble des émissions et que l'on mobilisera de plus en plus d'archives de télévision sous des formes diverses et variées. 